\title{
Bäcklund Transformations between the KdV Equation and a New Nonlinear Evolution Equation
}

\begin{abstract}
Xifang Cao
School of Mathematical Sciences, Yangzhou University, Yangzhou, China

Correspondence should be addressed to Xifang Cao; xfcao@yzu.edu.cn

Received 10 January 2017; Accepted 19 February 2017; Published 2 March 2017

Academic Editor: Alessandro Arsie

Copyright (C) 2017 Xifang Cao. This is an open access article distributed under the Creative Commons Attribution License, which permits unrestricted use, distribution, and reproduction in any medium, provided the original work is properly cited.

We first give a Bäcklund transformation from the KdV equation to a new nonlinear evolution equation. We then derive two Bäcklund transformations with two pseudopotentials, one of which is from the KdV equation to the new equation and the other from the new equation to itself. As applications, by applying our Bäcklund transformations to known solutions, we construct some novel solutions to the new equation.
\end{abstract}

\section{Introduction}

In 1895 two Dutch mathematicians Korteweg and de Vries derived a nonlinear wave equation which is now called $\mathrm{KdV}$ equation and adopts the canonical form [1]

$$
u_{t}=6 u u_{x}+u_{x x x} .
$$

Since the KdV equation (1) possesses the solitary wave solution

$$
u(x, t)=2 \kappa^{2} \operatorname{sech}^{2}\left(\kappa\left(x+4 \kappa^{2} t-x_{0}\right)\right),
$$

where $\kappa$ and $x_{0}$ are constants, it provides a theoretical confirmation of the existence of the solitary wave observed in 1834 by the Scottish engineer John Scott Russell on the Union canal. In 1965 Zabusky and Kruskal [2] discovered that the interaction of two solitary wave solutions is elastic, and therefore they called this kind of solutions solitons. In 1967 Gardner et al. [3] related the solution of the Cauchy initial value problem for the KdV equation (1) to the inverse scattering problem for a one-dimensional linear Schrödinger equation and derived the analytical expressions of $n$-soliton solutions. The $n$-soliton solutions to the KdV equation (1) can also be derived from the Darboux [4] and Bäcklund transformations [5], which have been extended and applied to a large variety of nonlinear evolution equations (see [613]).
It is well known that the $\mathrm{KdV}$ equation (1) is connected to the potential $\mathrm{KdV}$ equation

$$
v_{t}=3 v_{x}^{2}+v_{x x x}
$$

via $v=\int u \mathrm{~d} x$. By use of this connection, Wahlquist and Estabrook [14] obtained the following Bäcklund transformation for the $\mathrm{KdV}$ equation (1), as well as for the potential $\mathrm{KdV}$ equation (3).

Theorem 1 (see [14]). If $v$ is a solution of the potential KdV equation (3), then the system

$$
\begin{aligned}
v_{x}^{\prime}= & \lambda-v_{x}-\frac{1}{2}\left(v^{\prime}-v\right)^{2}, \\
v_{t}^{\prime}= & 2 \lambda^{2}-2 \lambda v_{x}-v_{x}^{2}-v_{x x x}+2 v_{x x}\left(v^{\prime}-v\right) \\
& -\left(\lambda+v_{x}\right)\left(v^{\prime}-v\right)^{2}
\end{aligned}
$$

on $v^{\prime}$ is integrable, where $\lambda$ is an arbitrary constant. Moreover, $v^{\prime}$ also satisfies (3). So the integrable system (4) defines a Bäcklund transformation $v \mapsto v^{\prime}$ for the potential KdV equation (3), and it also gives a Bäcklund transformation $u \mapsto$ $u^{\prime}$ for the KdV equation (1) which is defined by

$$
u \longmapsto v=\int u \mathrm{~d} x \longmapsto v^{\prime} \longmapsto u^{\prime}=v_{x}^{\prime} .
$$


Note that (4) is a Lax pair of the potential KdV equation (3). In this paper, we first give another Lax pair of (3) and show that it defines a Bäcklund transformation from the $\mathrm{KdV}$ equation (1) to a new nonlinear evolution equation (7) (see Theorem 2). Then by combining the Bäcklund transformations given in Theorems 1 and 2, we derive two Bäcklund transformations defined by two pseudopotentials (see Theorem 8): one is from (1) to (7) (see (22)) and the other from (7) to itself (see (23)). As applications, by applying our Bäcklund transformations to known solutions of (1), (3), or (7), we construct some novel solutions to (7) (see Examples $6,7,11$, and 12).

\section{Bäcklund Transformation with One Pseudopotential}

In this section, we prove the following result.

Theorem 2. If $v$ is a solution of (3), then the system on $\phi$

$$
\begin{aligned}
& \phi_{x}=(\phi+v)^{2} \\
& \phi_{t}=-v_{x}^{2}+2 v_{x x}(\phi+v)+2 v_{x}(\phi+v)^{2}
\end{aligned}
$$

is integrable. Moreover, $w=(\phi+v)^{2}$ satisfies the nonlinear evolution equation

$$
w^{2} w_{t}+6 w^{3} w_{x}-\frac{3}{4} w_{x}^{3}+\frac{3}{2} w w_{x} w_{x x}-w^{2} w_{x x x}=0
$$

Proof. From (3) and (6) we have

$$
\begin{aligned}
\phi_{x t} & =2(\phi+v) \\
\cdot & \left(-v_{x}^{2}+2 v_{x x}(\phi+v)+2 v_{x}(\phi+v)^{2}+3 v_{x}^{2}+v_{x x x}\right), \\
\phi_{t x} & =-2 v_{x} v_{x x}+2 v_{x x x}(\phi+v)+2 v_{x x}(\phi+v)^{2} \\
& +\left(2 v_{x x}+4 v_{x}(\phi+v)\right)\left((\phi+v)^{2}+v_{x}\right) .
\end{aligned}
$$

Therefore $\phi_{x t}=\phi_{t x}$; that is, (6) is integrable.

From the first equation of (6) we have

$$
\begin{aligned}
v & =\phi_{x}^{1 / 2}-\phi \\
v_{x} & =\frac{1}{2} \phi_{x}^{-1 / 2} \phi_{x x}-\phi_{x} \\
v_{x x} & =-\frac{1}{4} \phi_{x}^{-3 / 2} \phi_{x x}^{2}+\frac{1}{2} \phi_{x}^{-1 / 2} \phi_{x x x}-\phi_{x x} .
\end{aligned}
$$

Substituting the above equations into the second equation of (6) yields that $\phi$ satisfies

$$
\phi_{t}=-3 \phi_{x}^{2}-\frac{3}{4} \phi_{x}^{-1} \phi_{x x}^{2}+\phi_{x x x}
$$

and therefore

$$
\phi_{x t}=-6 \phi_{x} \phi_{x x}+\frac{3}{4} \phi_{x}^{-2} \phi_{x x}^{3}-\frac{3}{2} \phi_{x}^{-1} \phi_{x x} \phi_{x x x}+\phi_{x x x x}
$$

So $w=(\phi+v)^{2}=\phi_{x}$ satisfies (7).

To our knowledge, (7) has not been reported in literature previously.

Remark 3. If $w$ is a solution of (7), then

$$
v=w^{1 / 2}-\int w \mathrm{~d} x
$$

is a solution of (3).

Remark 4. The integrable system (6) gives a Bäcklund transformation from the potential $\mathrm{KdV}$ equation (3) to (7)

$$
v \longmapsto w=(\phi+v)^{2},
$$

which is defined by the pseudopotential $\phi[15]$.

Remark 5. The integrable system (6) also defines a Bäcklund transformation with the pseudopotential $\phi$ :

$$
u \longmapsto v=\int u \mathrm{~d} x \longmapsto w=(\phi+v)^{2}
$$

which takes a solution $u$ of the $\mathrm{KdV}$ equation (1) to a solution $w$ of (7).

Substituting a known solution $v$ of (3) into the integrable system (6), one can get a solution $w=(\phi+v)^{2}$ of (7). In the next two examples, from stationary and kink solutions of (3), we generate solutions of (7).

Example 6. Substituting the stationary solution of the potential $\mathrm{KdV}$ equation (3)

$$
v(x, t)=\frac{2}{x}
$$

into the integrable system (6) yields the following rational solution of (6),

$$
\phi(x, t)=-\frac{4 x^{3}-12 t}{x^{4}-12 x t}
$$

and therefore of (7)

$$
w(x, t)=\frac{4\left(x^{3}+6 t\right)^{2}}{x^{2}\left(x^{3}-12 t\right)^{2}} .
$$

The profiles of the solution (17) at $t=-1,0,1$ are shown in Figure 1 . Note that, at $t \neq 0$, the solution curve has three pieces; as $t$ approaches 0 , the piece located in the middle becomes smaller and smaller and finally disappears.

Example 7. Substituting the kink solution (see Figure 2) of the potential $\mathrm{KdV}$ equation (3)

$$
v(x, t)=2 k \tanh \left(k x+4 k^{3} t\right),
$$



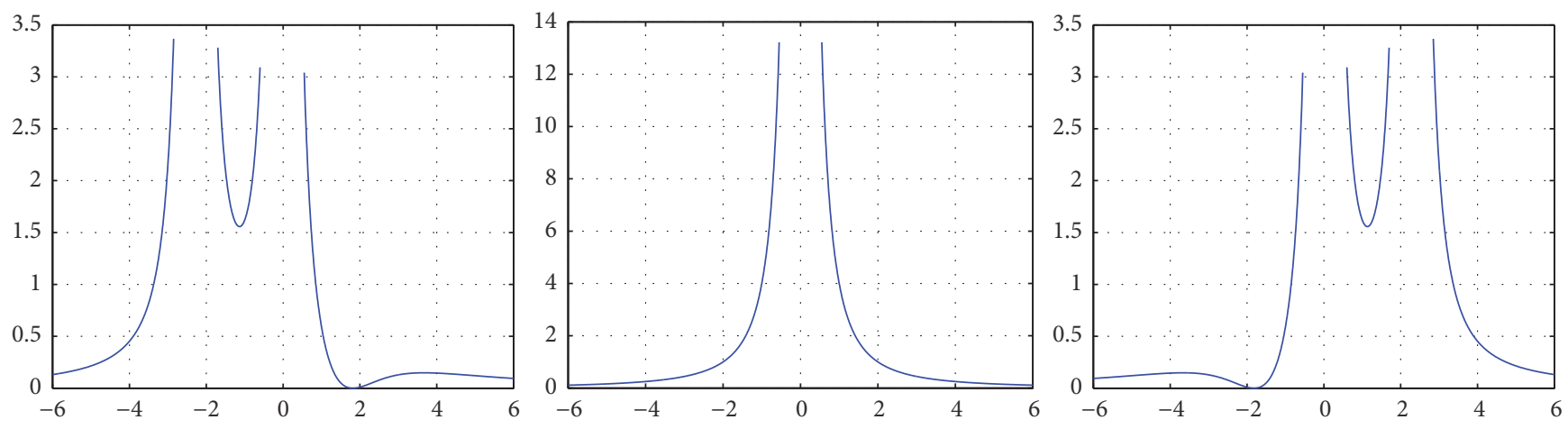

FIGURE 1: Profiles of solution (17).

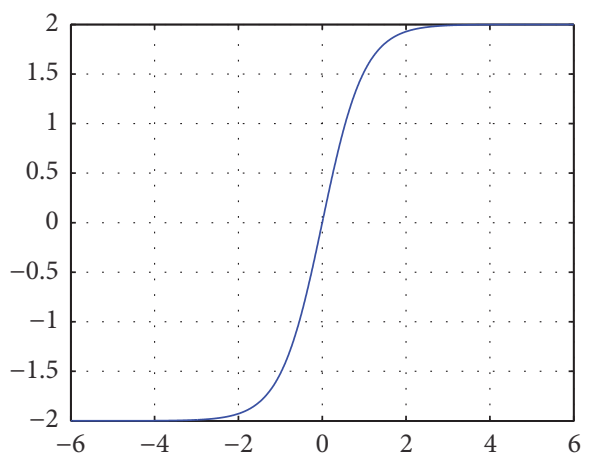

FIGURE 2: Profile of kink solution (18).

(where $k$ is an arbitrary nonzero constant) into the integrable system (6) yields the following solution of (6),

$$
\phi(\mathrm{x}, t)=-k\left(\tanh \left(k x+4 k^{3} t\right)+\operatorname{coth}\left(k x+4 k^{3} t\right)\right),
$$

and therefore of (7)

$$
\begin{aligned}
& w(x, t) \\
& \quad=k^{2}\left(\tanh \left(k x+4 k^{3} t\right)-\operatorname{coth}\left(k x+4 k^{3} t\right)\right)^{2} .
\end{aligned}
$$

Figure 3 illustrates the wave form of the solution (20) for $k=1$.

\section{Bäcklund Transformations with Two Pseudopotentials}

By combining Theorems 1 and 2, we have the following result. The proof is obvious (see, for example, [16]).

Theorem 8. If $v$ is a solution of (3), then the system on $\theta$ and $\phi$

$$
\begin{aligned}
& \theta_{x}=\lambda-v_{x}-\frac{1}{2}(\theta-v)^{2}, \\
& \phi_{x}=(\theta+\phi)^{2},
\end{aligned}
$$

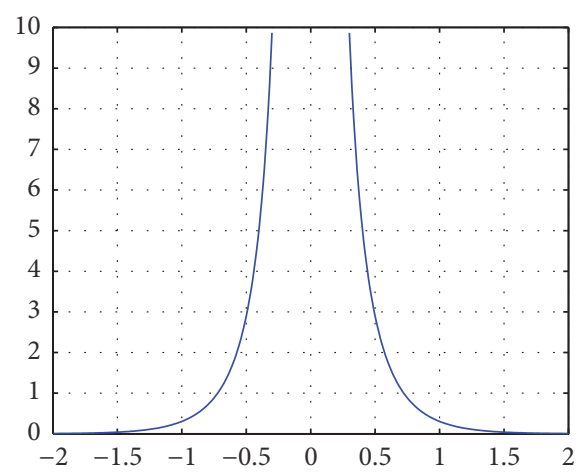

FIGURE 3: Profile of solution (20).

$$
\begin{aligned}
\theta_{t}= & 2 \lambda^{2}-2 \lambda v_{x}-v_{x}^{2}-v_{x x x}+2 v_{x x}(\theta-v) \\
& -\left(\lambda+v_{x}\right)(\theta-v)^{2}, \\
\phi_{t}= & -\theta_{x}^{2}+2 \theta_{x x}(\theta+\phi)+2 \theta_{x}(\theta+\phi)^{2}
\end{aligned}
$$

is integrable, where $\lambda$ is an arbitrary constant. Moreover, $w=$ $(\theta+\phi)^{2}$ satisfies (7).

Remark 9. The integrable system (21) gives a Bäcklund transformation $u \mapsto w$ from the KdV equation (1) to (7) as follows:

$$
u \longmapsto v=\int u \mathrm{~d} x \longmapsto w=(\theta+\phi)^{2}
$$

which is defined by two pseudopotentials $\theta$ and $\phi$ [15].

Remark 10. The integrable system (21) also defines a Bäcklund transformation $w \mapsto w^{\prime}$ with two pseudopotentials $\theta$ and $\phi$ for (7) as follows:

$$
w \longmapsto v=w^{1 / 2}-\int w \mathrm{~d} x \longmapsto w^{\prime}=(\theta+\phi)^{2} .
$$




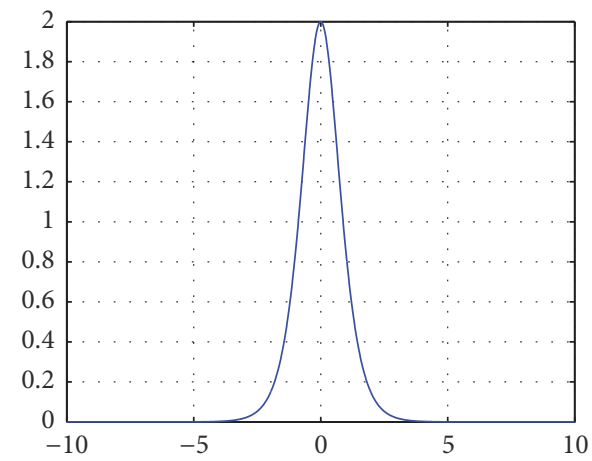

FIgURe 4: Profile of one-soliton (24).
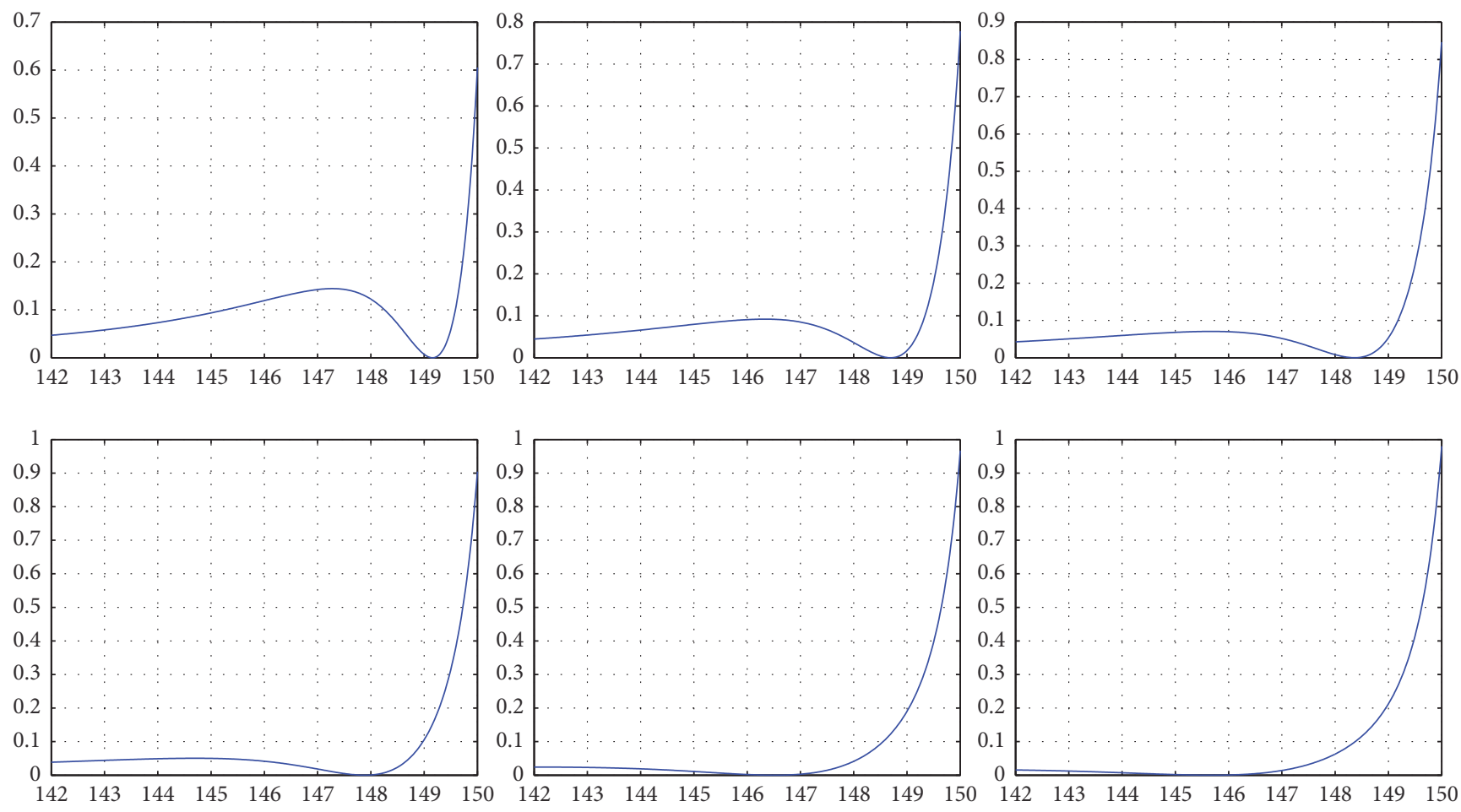

FIGURE 5: Profiles of $w$ generated by applying Bäcklund transformation (22) to one-soliton (24).

Applying the Bäcklund transformation (22) or (23) to a known solution $u$ of (1) or $w$ of (7), it is usually not easy to get explicit formulas for $\theta$ and $\phi$ when solving the integrable system (21). But in this case, one can use an ordinary differential equation solver (see, for example, [13, 16]) to obtain numerical approximations of $\theta$ and $\phi$. In the next two examples, we use this method.

Example 11. Applying the Bäcklund transformation (22) to the one-soliton solution (see Figure 4)

$$
u(x, t)=2 k^{2} \cosh ^{-2}\left(k x+4 k^{3} t\right)
$$

of the $\mathrm{KdV}$ equation (1), where $k$ is an arbitrary nonzero constant, we generate solution $w$ of (7). Figure 5 consists of the wave forms of $w$ for $\lambda=0$ with initial values $\theta(150,0)=0$ and $\phi(150,0)=0$ at $t=1,2,3$ (first row) and at $t=5,15,25$ (second row), which shows the evolution with time of this solution.

Example 12. Applying the Bäcklund transformation (23) to the rational solution (17) of (7), we generate new solution $w^{\prime}$ of (7). Figure 6 shows the profiles of $w^{\prime}$ for $\lambda=0$ with initial values $\theta(150,0)=-1$ and $\phi(150,0)=1$ at $t=1,2,3$ (first row), at $t=5,10,20$ (second row), and at $t=30,40,50$ (third row), which describes the evolution with time of this solution. 

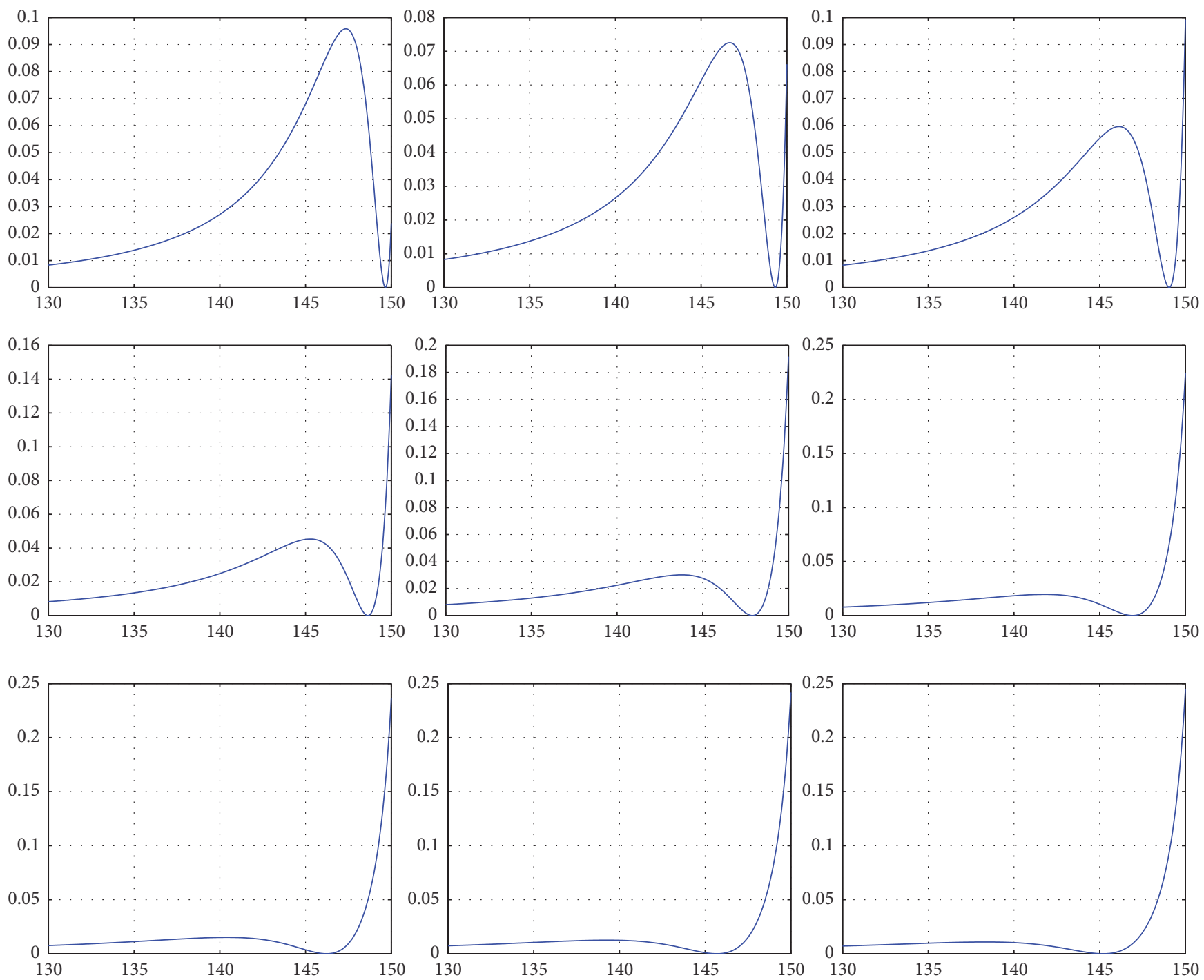

FIGURE 6: Profiles of $w^{\prime}$ generated by applying Bäcklund transformation (23) to rational solution (17).

\section{Competing Interests}

The author declares that they have no competing interests.

\section{Acknowledgments}

This work is supported by the National Natural Science Foundation of China through 11571299 and the Natural Science Foundation of Jiangsu Province through BK20151304. The author would like to thank Dr. Lai Zhang from Umeå University for his help of plotting the figures.

\section{References}

[1] M. J. Ablowitz and P. A. Clarkson, Solitons, nonlinear evolution equations and inverse scattering, vol. 149 of London Mathematical Society Lecture Note Series, Cambridge University Press, Cambridge, UK, 1991.
[2] N. J. Zabusky and M. D. Kruskal, "Interaction of 'solitons' in a collisionless plasma and the recurrence of initial states," Physical Review Letters, vol. 15, no. 6, pp. 240-243, 1965.

[3] C. S. Gardner, J. M. Greene, M. D. Kruskal, and R. M. Miura, "Method for Solving the Korteweg-deVries Equation," Physical Review Letters, vol. 19, no. 19, pp. 1095-1097, 1967.

[4] V. B. Matveen and M. A. Salle, Darboux Transformations and Solitons, Springer, Berlin, Germany, 1991.

[5] C. Rogers and W. K. Schief, Bäcklund and Darboux Transformations. Geometry and Modern Applications in Soliton Theory, Cambridge University Press, Cambridge, UK, 2002.

[6] C. Rogers and W. F. Shadwick, Bäcklund Transformations and Their Applications, Academic Press, New York, NY, USA, 1982.

[7] R. M. Miura, "Korteweg-de Vries equation and generalizations. I. A remarkable explicit nonlinear transformation," Journal of Mathematical Physics, vol. 9, pp. 1202-1204, 1968.

[8] G. L. Lamb Jr., "Bäcklund transformations for certain nonlinear evolution equations," Journal of Mathematical Physics, vol. 15, pp. $2157-2165,1974$ 
[9] S. I. Svinolupov, V. V. Sokolov, and R. I. Yamilov, "Bäcklund transformations for integrable evolution equations," Doklady Akademii Nauk SSSR, vol. 271, pp. 802-805, 1983 (Russian).

[10] K. Tenenblat, Transformations of manifolds and applications to differential equations, vol. 93 of Pitman Monographs and Surveys in Pure and Applied Mathematics, Longman, Harlow, UK, 1998.

[11] T. Chou, "Bäcklund transformation of nonlinear evolution equations," Acta Mathematicae Applicatae Sinica, vol. 2, no. 1, pp. 87-94, 1985.

[12] H. Wu, "On Bäcklund transformations for nonlinear partial differential equations," Journal of Mathematical Analysis and Applications, vol. 192, no. 1, pp. 151-179, 1995.

[13] X. Cao, "Bäcklund transformations with two pseudo-potential," Applied Mathematics Letters, vol. 61, pp. 13-19, 2016.

[14] H. D. Wahlquist and F. B. Estabrook, "Bäcklund transformation for solutions of the Korteweg-de Vries equation," Physical Review Letters, vol. 31, pp. 1386-1390, 1973.

[15] H. D. Wahlquist and F. B. Estabrook, "Prolongation structures of nonlinear evolution equations," Journal of Mathematical Physics, vol. 16, pp. 1-7, 1975.

[16] X. Cao, C. Xu, and H. Wu, "On Miura transformations among nonlinear partial differential equations," Journal of Mathematical Physics, vol. 47, no. 8, Article ID 083515, 2006. 


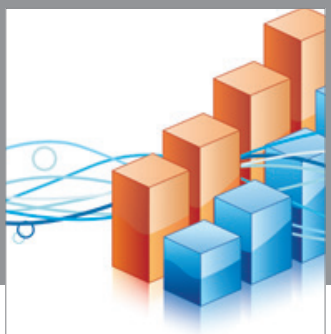

Advances in

Operations Research

vatem alat4

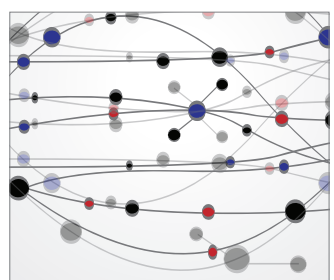

\section{The Scientific} World Journal
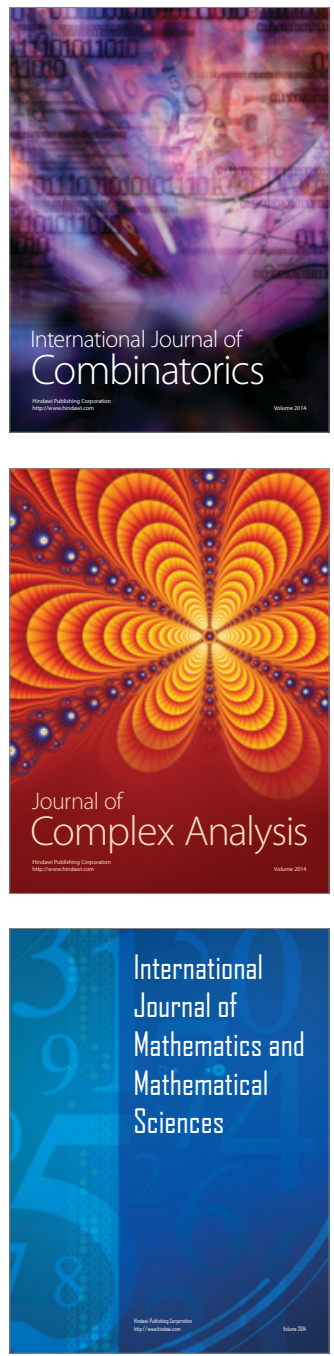
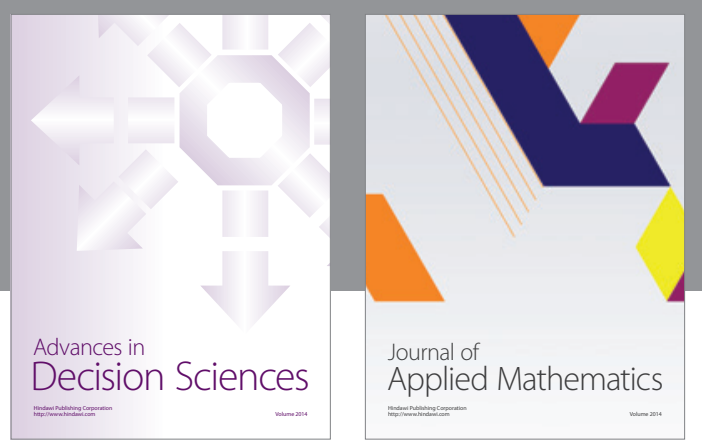

Algebra

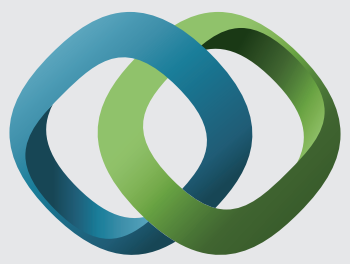

\section{Hindawi}

Submit your manuscripts at

https://www.hindawi.com
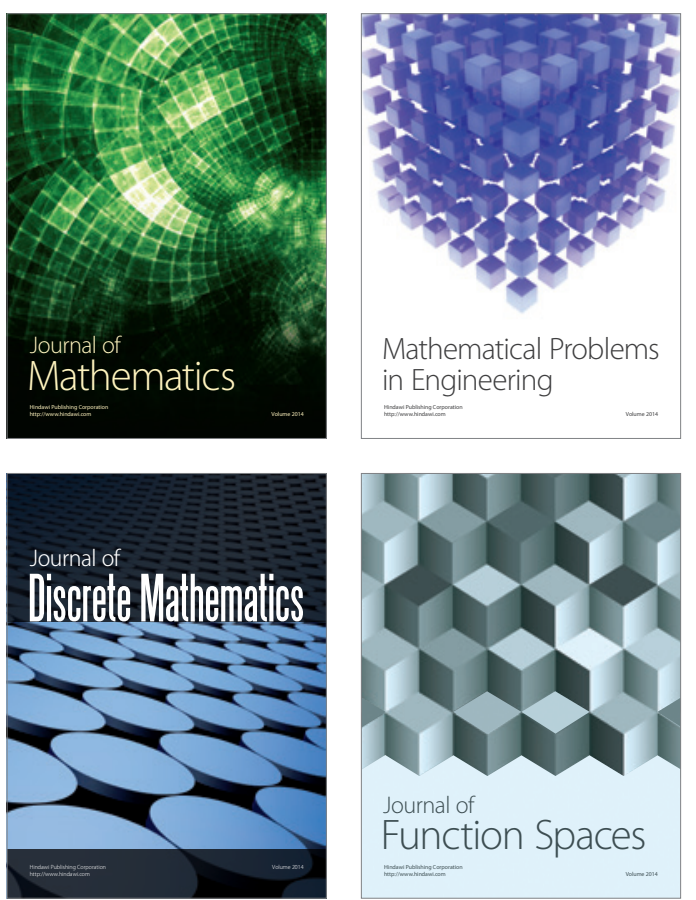

Mathematical Problems in Engineering
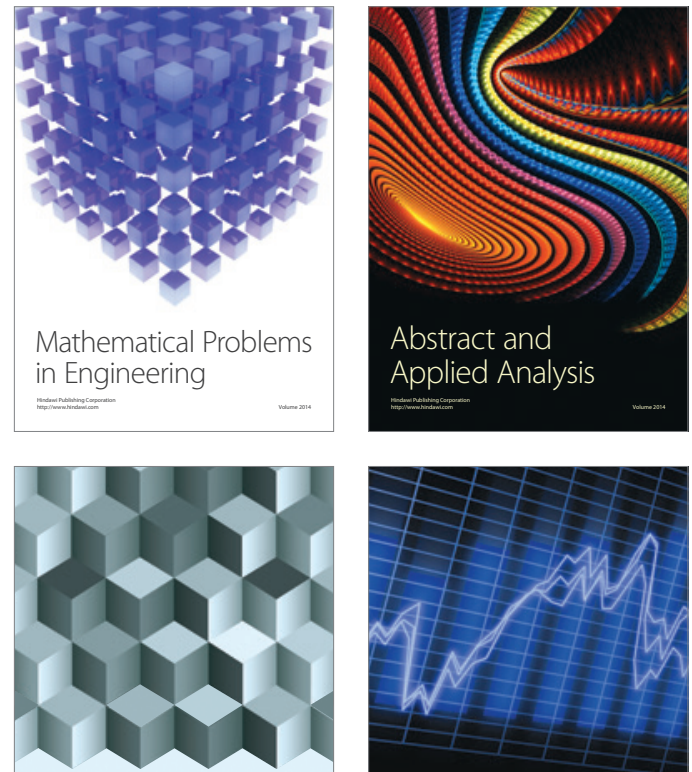

Journal of

Function Spaces

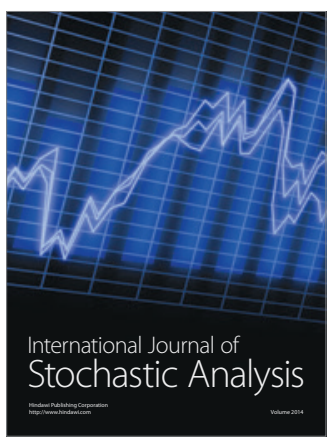

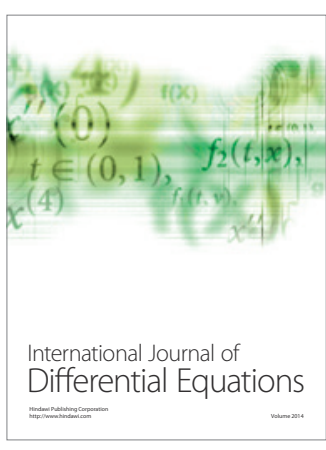
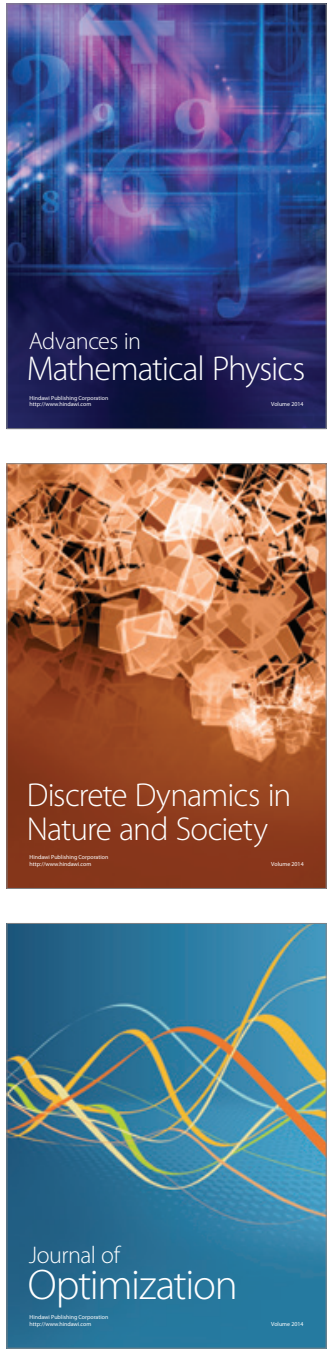